\title{
Dietary patterns and the risk of non-Hodgkin lymphoma
}

\author{
Nicholas J Ollberding ${ }^{1,2, *}$, Briseis Aschebrook-Kilfoy ${ }^{2}$, Donne Bennett D Caces ${ }^{3}$, \\ Sonali M Smith ${ }^{3}$, Dennis D Weisenburger ${ }^{4}$ and Brian C-H Chiv ${ }^{2,5}$ \\ 'Division of Biostatistics and Epidemiology, Cincinnati Children's Hospital Medical Center, Cincinnati, OH, USA: \\ ${ }^{2}$ Department of Health Studies, University of Chicago, 5841 South Maryland Avenue, MC 2007, Chicago, \\ IL 60637, USA: ${ }^{3}$ Division of Hematology/Oncology, Department of Medicine, University of Chicago, Chicago, \\ IL, USA: ${ }^{4}$ Department of Pathology and Microbiology, University of Nebraska Medical Center, Omaha, NE, \\ USA: ${ }^{5}$ University of Chicago Comprehensive Cancer Center, Chicago, IL, USA
}

Submitted 17 October 2012: Final revision received 19 February 2013: Accepted 4 April 2013: First published online 9 May 2013

\begin{abstract}
Objective: Previous studies examining the role of single foods or nutrients in the aetiology of non-Hodgkin lymphoma (NHL) have produced inconsistent findings. Few studies have examined associations for dietary patterns, which may more accurately reflect patterns of consumption and the complexity of dietary intake. The objective of the present study was to examine whether dietary patterns identified by factor analysis were associated with NHL risk.

Design: Case-control.

Setting: Population-based sample residing in Nebraska from 1999 to 2002.

Subjects: A total of 336 cases and 460 controls.

Results: Factor analysis identified two major dietary patterns: (i) a 'Meat, Fat and Sweets' dietary pattern characterized by high intakes of French fries, red meat, processed meat, pizza, salty snacks, sweets and desserts, and sweetened beverages; and (ii) a 'Fruit, Vegetables and Starch' dietary pattern characterized by high intakes of vegetables, fruit, fish, and cereals and starches. In multivariable logistic regression models, the 'Meat, Fat and Sweets' dietary pattern was associated with an increased risk of overall NHL $\left(\mathrm{OR}_{\mathrm{Q} 4 v \cdot \mathrm{Q} 1}=3 \cdot 6\right.$, $95 \%$ CI $\left.1 \cdot 9,6 \cdot 8 ; P_{\text {trend }}=0 \cdot 0004\right)$, follicular lymphoma $\left(\mathrm{OR}_{\mathrm{Q} 4 v \cdot \mathrm{Q} 1}=3 \cdot 1,95 \%\right.$ CI $\left.1 \cdot 2,8 \cdot 0 ; \quad P_{\text {trend }}=0 \cdot 01\right)$, diffuse large B-cell lymphoma $\left(\mathrm{OR}_{\mathrm{Q} 4 v \cdot \mathrm{Q} 1}=3 \cdot 2\right.$, $95 \%$ CI $\left.1 \cdot 1,9 \cdot 0 ; P_{\text {trend }}=0 \cdot 09\right)$ and marginal zone lymphoma $\left(\mathrm{OR}_{\mathrm{Q} 4} v \cdot \mathrm{Q} 1=8 \cdot 2\right.$, $95 \%$ CI $\left.1 \cdot 3,51 \cdot 2 ; P_{\text {trend }}=0 \cdot 05\right)$. No association with overall or subtype-specific risk was detected for the 'Fruit, Vegetables and Starch' dietary pattern. No evidence of heterogeneity was detected across strata of age, sex, BMI, smoking status or alcohol consumption.

Conclusions: Our results suggest that a dietary pattern high in meats, fats and sweets may be associated with an increased risk of NHL.
\end{abstract}

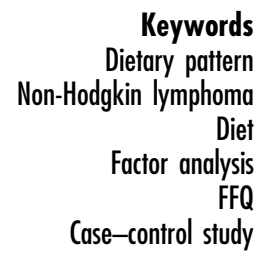

The non-Hodgkin lymphomas (NHL) are a heterogeneous group of malignant neoplasms arising from the $\mathrm{B}, \mathrm{T}$ and natural killer cells of the immune system. NHL is the fifth most commonly diagnosed cancer in the USA ${ }^{(1)}$, with immune dysregulation thought to contribute to lymphoma development ${ }^{(2,3)}$. However, well-characterized immunodeficiency states only partially account for the rising rates of this disease in recent decades ${ }^{(3,4)}$. Dietary factors have been examined in relation to the development of NHL given their role in immune system regulation, oxidative stress and hormonal pathways regulating the proliferation of lymphoid tissue ${ }^{(5)}$.

Previous studies examining the association between single food items and the risk of NHL have reported inconsistent findings for items such as red meat and processed meat, fruits and vegetables, and dairy products ${ }^{(5,6)}$.
These inconsistencies may, in part, reflect the difficulty in disentangling the influence of individual food items that, when consumed in combination, may be highly correlated and exert synergistic or antagonistic effects on NHL risk. The examination of dietary patterns, which better reflect actual patterns of consumption and the complexity of dietary intake, has been used to address such limitations ${ }^{(7)}$. Factor analysis is an approach that can be applied to dietary data to identify underlying patterns of dietary intake based on the intercorrelations of individual foods and food groups.

In the present study, we used factor analysis to identify empirically derived dietary patterns in a population of adults residing in eastern Nebraska and to examine the associations of these dietary patterns with the risk of overall NHL. In secondary analyses, we examined the associations with risk for the common NHL subtypes. 


\section{Materials and methods}

\section{Study population}

Detailed information on the present case-control study has been reported previously ${ }^{(8,9)}$. Briefly, a rapid case ascertainment system was used to identify cases of NHL diagnosed in the sixty-six counties of eastern Nebraska between January 1999 and December 2002. All cases were reviewed and classified by an expert haematopathologist (D.D.W.) in accordance with the WHO classification of $\mathrm{NHL}^{(10)}$. Persons reporting any prior cancer (other than cutaneous squamous cell carcinoma or basal cell carcinoma), HIV infection, were deceased at initial contact or not mentally competent to participate were not included. Of the 529 eligible cases, 387 (73\%) participated in the study. The controls were frequency matched to the cases on gender and 5-year age group and were identified through random digit dialling of the same sixty-six counties in eastern Nebraska. Of the 697 eligible controls, $535(77 \%)$ participated in the study. Informed consent was obtained from all participants prior to the interview and the study protocol was approved by the Institutional Review Board of the University of Nebraska Medical Center.

\section{Dietary assessment}

A structured telephone interview, administered concurrently for cases and for controls, was used to collect information on demographic characteristics, lifestyle factors and environmental exposures. Following the administration of the telephone interview, a modified version of the Block 1995 Revision of the Health Habits and History Questionnaire (HHHQ) was mailed to all participants ${ }^{(11)}$. This quantitative FFQ included queries on 117 items and assessed the consumption of food items during the previous year. The HHHQ was developed using dietary data from the National Health and Nutrition Examination Survey II ${ }^{(12)}$ and has been validated against dietary records with correlation coefficients in the range of $0 \cdot 5-0 \cdot 6$ for most nutrients ${ }^{(13)}$. The FFQ was completed and returned by 348 (90\%) cases and 470 (88\%) controls. The participants who did not return the FFQ were slightly younger, but were similar with respect to BMI, smoking status and educational attainment to participants who returned the FFQ. In addition, excluded from the current analysis were twelve cases and ten controls who reported total energy intake of $<3347$ or $>25105 \mathrm{~kJ} / \mathrm{d}$ $(<800$ or $>6000 \mathrm{kcal} / \mathrm{d})$ for men and $<2510$ or $>20920 \mathrm{~kJ} / \mathrm{d}$ $(<600$ or $>5000 \mathrm{kcal} / \mathrm{d}$ ) for women or left more than $20 \%$ of the items blank on the FFQ. In total, 336 cases and 460 controls provided complete dietary data and were included in the present analysis.

Prior to conducting the dietary pattern factor analysis, we aggregated the 117 food items queried by the FFQ into thirty-seven predefined food groups (Table 1). The food groups were classified according to similarities in food type, nutrient content and culinary usage. Food items representing distinct food types (e.g. pizza, French fries, beer, liquor) were retained as individual items for the factor analysis. A factor analysis deriving dietary patterns from all 117 food items was also performed to assess the influence of the classification on the dietary patterns identified.

\section{Statistical analysis}

Prior to performing the factor analysis, the factorability of the data was supported by examination of the correlation matrix for the thirty-seven foods and food groups, Bartlett's test of sphericity $(P<0 \cdot 001)$ rejecting the null hypothesis that the correlation matrix is from an identity matrix and the Kaiser-Meyer-Olkin measure of sampling adequacy (MSA $=0 \cdot 82$ ) indicating sufficient partial correlations among variables. Exploratory factor analysis with principal component factor extraction was used to identify latent factors (dietary patterns) explaining the greatest amount of variance in the correlation matrix. The factors were rotated by orthogonal varimax transformation to obtain independent factors with a simpler structure and greater interpretability. The number of factors chosen to retain was based on the following criteria: eigenvalue $>1$, visual examination of the scree plot and the interpretability of the factors $^{(14)}$. Individual factor scores for each dietary pattern were calculated by weighted least squares regression. Foods and food groups with a factor loading $\geq 0 \cdot 30$ were used to label the factors.

Characteristics of the cases and of the controls were compared using $\chi^{2}$ tests for categorical variables and $t$ tests for continuous variables. Unconditional logistic regression was used to calculate odds ratios and 95\% confidence intervals for overall NHL. Polytomous logistic regression was used to calculate odds ratios and 95\% confidence intervals for six common subtypes of NHL according to the WHO classification: follicular lymphoma (FL), diffuse large B-cell lymphoma (DLBCL), small lymphocytic lymphoma/chronic lymphocytic leukaemia (SLL/CLL), marginal zone lymphoma (MZL), other miscellaneous B-cell lymphomas and T-cell lymphomas. Dietary pattern scores were examined as quartiles using cut-off points based on the exposure distribution among controls. The lowest exposure group (quartile 1) served as the referent in all models. Linear trends were tested by entering quartiles as ordinal variables in regression models. Models were also constructed to examine the associations between dietary pattern scores entered into regression as continuous variables and NHL risk. Potential non-linear relationships were first assessed by a likelihood ratio test comparing the model with only the linear term to a model containing linear and cubic spline terms. Covariates included in the final multivariable models were age (continuous), sex, education $(<12$ years, $12-15$ years, $16+$ years) and total energy intake (continuous). Alcohol consumption, smoking status, BMI, physical activity, farming status, use of hair dye and history of blood transfusion were examined as potential confounders, but were not 
Table 1 Classification of food groups used in the dietary pattern factor analysis

\begin{tabular}{|c|c|}
\hline Foods or food groups & Food items \\
\hline Red meat & Beef, pork, lamb, hamburger \\
\hline Poultry & Chicken, turkey \\
\hline Processed meat & Bacon, hot dogs, sausage, processed lunch meat \\
\hline Organ meat & Beef or poultry liver \\
\hline Fish & Broiled fish, fried fish, canned tuna, shellfish \\
\hline High-fat dairy products & Cream, cottage cheese, yoghurt, cheese, ice cream, whole milk \\
\hline Low-fat dairy products & Low-fat cheese, low-fat cottage cheese, low-fat ice cream, low-fat yoghurt, $2 \%$ milk, skimmed milk \\
\hline Cruciferous vegetables & Broccoli, cauliflower, Brussels sprouts, cabbage \\
\hline Green leafy vegetables & Spinach, green salad, collards/kale \\
\hline Legumes and soya & Alfalfa sprouts, lentils, peas, string beans, tofu, meat substitutes \\
\hline Potatoes & Baked/mashed potatoes, sweet potatoes \\
\hline Tomatoes or tomato juice & Tomatoes or tomato juice \\
\hline Other vegetables & Rhubarb, beets, radishes, celery, carrots, corn, other vegetables \\
\hline Vegetable soup & Vegetable soup \\
\hline Other soups & Other soups \\
\hline Fruits and fruit juice & $\begin{array}{l}\text { Oranges/tangerines, orange juice, apple/grape juice, watermelon, grapefruit, strawberries, } \\
\text { prunes, peaches/apricots, apples, bananas, other fruits }\end{array}$ \\
\hline Nuts & Peanuts/peanut butter \\
\hline Cereals and starches & $\begin{array}{l}\text { Cornbread, biscuits/muffins, dry cereals, sweetened cereals, high-fibre cereals, bagels/English } \\
\text { muffins/buns, dark bread, cooked cereal/grits, white bread, tortillas, pancakes/waffles }\end{array}$ \\
\hline Pasta and rice & Pasta salad, macaroni, rice, spaghetti \\
\hline Pizza & Pizza \\
\hline French fries & French fries \\
\hline Salty snacks & Salty snacks, nachos \\
\hline Sweets and desserts & Cookies/cakes, pies, candy, chocolate, doughnuts/pastry \\
\hline Eggs & Eggs, egg substitutes \\
\hline Butter & Butter, whipped butter \\
\hline Margarine & Margarine \\
\hline Cooking fat and gravy & Crisco, lard, gravy \\
\hline Olive oil or vegetable oil & Olive/canola oil, vegetable oil \\
\hline Mayonnaise or creamy salad dressing & Mayonnaise/salad dressing \\
\hline Condiments & Salsa/ketchup/taco sauce, sugar in coffee or tea, non-dairy creamer \\
\hline Meal replacements & Breakfast or diet shakes, breakfast bars/power bars \\
\hline Wine & Red wine, white wine \\
\hline Beer & Beer \\
\hline Liquor & Liquor \\
\hline Sweetened beverages & Soft drinks, Kool-Aid or similar, Snapple or similar \\
\hline Coffee or tea & Coffee, tea \\
\hline Water & Water \\
\hline
\end{tabular}

Food items were measured in $\mathrm{g} / \mathrm{d}$ using a modified version of the Block 1995 Revision of the Health Habits and History Questionnaire ${ }^{(11)}$.

included in the final models as they were not found alone, or in combination, to change the risk estimates by more than $10 \%{ }^{(15)}$. In addition, mutual adjustment for the dietary patterns was performed.

The associations for the dietary patterns with overall NHL risk were also examined in analyses stratified by sex, age $\left(<61\right.$ years $v$. $\geq 61$ years, median), BMI $\left(<25 \mathrm{~kg} / \mathrm{m}^{2}\right.$, $25-<30 \mathrm{~kg} / \mathrm{m}^{2}, \geq 30 \mathrm{~kg} / \mathrm{m}^{2}$ ), smoking status (never smoker, former smoker, current smoker), alcohol consumption $(<0.3 \%$ of energy/d $v . \geq 0 \cdot 3 \%$ of energy/d, median) and NHL subtype. Heterogeneity in the risk estimates by sex, age, BMI, smoking status and alcohol consumption was assessed using a Wald test of the cross-product terms. Heterogeneity in the risk estimates across the six NHL subtypes was assessed using a Wald test of the parameter estimates obtained from unconditional polytomous logistic regression comparing case subgroups. All tests were twosided with $\alpha$ of $P<0.05$ considered statistically significant. Data analyses were performed using the statistical software package SAS version $9 \cdot 2$.

\section{Results}

Selected characteristics of the cases and of the controls are given in Table 2. Cases and controls were similar with respect to age, sex, race, educational attainment, firstdegree family history of cancer, smoking status, alcohol consumption, BMI and energy intake. The majority of NHL cases were of B-cell origin (>94\%) and classified as SLL/CLL (7·4\%), FL (31·3\%), DLBCL (26.5\%), MZL (8.9\%) or other B-cell lymphomas $(20 \cdot 2 \%)$, with only a small number of T-cell lymphomas $(5 \cdot 7 \%)$.

The principal component factor extraction identified two dietary patterns that were labelled descriptively as a 'Meat, Fat and Sweets' dietary pattern and a 'Fruit, Vegetables and Starch' dietary pattern based on factor score loadings (Table 3). The 'Meat, Fat and Sweets' dietary pattern loaded high on French fries, red meat, processed meat, pizza, salty snacks, sweets and desserts, sweetened beverages, condiments, cooking fat and gravy, margarine, high-fat dairy foods, pasta and rice, and eggs. The 'Fruit, 
Table 2 Characteristics of eligible non-Hodgkin lymphoma (NHL) cases and controls, Nebraska, USA, 1999-2002

\begin{tabular}{|c|c|c|c|c|c|}
\hline & \multicolumn{2}{|c|}{ Cases ( $n$ 336) } & \multicolumn{2}{|c|}{ Controls ( $n$ 460) } & \multirow[b]{2}{*}{$P$ value* } \\
\hline & Mean or $n$ & SD or $\%$ & Mean or $n$ & SD or $\%$ & \\
\hline Age (years) & $58 \cdot 6$ & $12 \cdot 7$ & $58 \cdot 0$ & $12 \cdot 8$ & 0.54 \\
\hline Malet & 185 & $55 \cdot 1$ & 236 & $51 \cdot 3$ & $0 \cdot 29$ \\
\hline White racet & 325 & $96 \cdot 7$ & 445 & $96 \cdot 7$ & 0.99 \\
\hline \multicolumn{6}{|l|}{ Educationt } \\
\hline$<12$ years & 15 & $4 \cdot 5$ & 9 & $2 \cdot 0$ & \\
\hline $12-15$ years & 126 & $37 \cdot 8$ & 191 & $41 \cdot 5$ & \\
\hline $16+$ years & 192 & $57 \cdot 7$ & 260 & $56 \cdot 5$ & 0.09 \\
\hline \multicolumn{6}{|l|}{ First-degree family history of cancert } \\
\hline None & 159 & $47 \cdot 3$ & 224 & $48 \cdot 7$ & \\
\hline Non-haematopoietic & 140 & $41 \cdot 7$ & 196 & $42 \cdot 6$ & \\
\hline Haematopoietic & 37 & $11 \cdot 0$ & 40 & $8 \cdot 7$ & 0.55 \\
\hline \multicolumn{6}{|l|}{ Smoking statust } \\
\hline Never & 169 & $52 \cdot 2$ & 221 & $50 \cdot 3$ & \\
\hline Former & 108 & $33 \cdot 3$ & 140 & $31 \cdot 9$ & \\
\hline Current & 47 & $14 \cdot 5$ & 78 & $17 \cdot 8$ & 0.48 \\
\hline \multicolumn{6}{|l|}{ BMI statust } \\
\hline Normal/underweight $\left(<25 \mathrm{~kg} / \mathrm{m}^{2}\right)$ & 103 & $30 \cdot 9$ & 160 & $34 \cdot 8$ & \\
\hline Overweight $\left(25\right.$ to $\left.<30 \mathrm{~kg} / \mathrm{m}^{2}\right)$ & 131 & $39 \cdot 3$ & 188 & $40 \cdot 9$ & \\
\hline Obese $\left(\geq 30 \mathrm{~kg} / \mathrm{m}^{2}\right)$ & 99 & $29 \cdot 7$ & 112 & $24 \cdot 3$ & $0 \cdot 21$ \\
\hline Percentage of energy from alcohol & $2 \cdot 2$ & $5 \cdot 2$ & $2 \cdot 6$ & $5 \cdot 7$ & 0.31 \\
\hline Energy $(\mathrm{kJ} / \mathrm{d})$ & 8303 & 3310 & 7996 & 2955 & $0 \cdot 18$ \\
\hline Energy $(\mathrm{kcal} / \mathrm{d})$ & $1984 \cdot 5$ & $791 \cdot 0$ & $1911 \cdot 1$ & $706 \cdot 3$ & $0 \cdot 18$ \\
\hline \multicolumn{6}{|l|}{ WHO-defined NHL subtypest } \\
\hline SLL/CLL & 25 & $7 \cdot 4$ & & & \\
\hline $\mathrm{FL}$ & 105 & $31 \cdot 3$ & & & \\
\hline DLBCL & 89 & $26 \cdot 5$ & & & \\
\hline MZL & 30 & $8 \cdot 9$ & & & \\
\hline Other B-cell lymphomas & 68 & $20 \cdot 2$ & & & \\
\hline T-cell lymphomas & 19 & $5 \cdot 7$ & & & \\
\hline
\end{tabular}

SLL/CLL, small lymphocytic lymphoma/chronic lymphocytic leukaemia; FL, follicular lymphoma; DLBCL, diffuse large B-cell lymphoma; MZL, marginal zone lymphoma.

Numbers may not sum to the total due to missing data.

${ }^{\star} P$ value for the $\chi^{2}$ test for categorical variables and the $t$ test for continuous variables when comparing the proportions and the means respectively between cases and controls.

tValues presented are $n$ and \%.

Vegetables and Starch' dietary pattern loaded high on other vegetables, tomatoes and juice, cruciferous vegetables, green leafy vegetables, fruit and fruit juice, legumes and soya, vegetable soup, fish, mayonnaise and creamy salad dressing, cereals and starches, potatoes, other soups, and low-fat dairy foods. Similar factors were identified when deriving dietary patterns from all 117 food items (data not shown).

The odds ratios and 95\% confidence intervals for NHL according to quartiles of the factor scores for the two dietary patterns are provided in Table 4. In models adjusting for age, sex, educational attainment and total energy, the 'Meat, Fat and Sweets' dietary pattern was associated with an increased risk of overall NHL $\left(\mathrm{OR}_{\mathrm{Q} 4} v \cdot \mathrm{Q} 1=3 \cdot 6,95 \% \mathrm{CI} 1 \cdot 9,6 \cdot 8 ; P_{\text {trend }}=0 \cdot 0004\right)$. The results were similar when the dietary pattern was entered into regression as a continuous variable (the log of the unit change in the odds ratio per unit change in the factor score $(\beta)=0.54, \mathrm{SE}=0.12 ; P<0 \cdot 0001)$. Red meat $(\beta=0.003$, SE $=0.001 ; \quad P=0 \cdot 01)$ and pizza $(\beta=0 \cdot 02$, SE $=0.006 ; \quad P=0.004)$ were the only individual food groups loading high on the 'Meat, Fat and Sweets' dietary pattern that demonstrated a linear association with the risk of overall NHL. No association with overall NHL risk was detected for the 'Fruit, Vegetables and Starch' dietary pattern when examined as quartiles of exposure $\left(\mathrm{OR}_{\mathrm{Q} 4 v . \mathrm{Q} 1}=0.9,95 \% \mathrm{CI} 0.6,1.4 ; P_{\text {trend }}=0.75\right)$ or as a continuous variable $(\beta=-0.05, \quad \mathrm{sE}=0.09 ; \quad P<0.56)$. In subtype-specific analyses, the 'Meat, Fat and Sweets' dietary pattern was associated with an increased risk of $\mathrm{FL}\left(\mathrm{OR}_{\mathrm{Q} 4 v \cdot \mathrm{Q} 1}=3 \cdot 1,95 \%\right.$ CI $\left.1 \cdot 2,8 \cdot 0 ; P_{\text {trend }}=0 \cdot 01\right)$, DLBCL $\left(\mathrm{OR}_{\mathrm{Q} 4} v \cdot \mathrm{Q} 1=3 \cdot 2,1 \cdot 1,9 \cdot 0 ; P_{\text {trend }}=0 \cdot 09\right)$ and MZL $\left(\mathrm{OR}_{\mathrm{Q} 4 v \cdot \mathrm{Q} 1}=8 \cdot 2, \quad 95 \%\right.$ CI $\left.1 \cdot 3,51 \cdot 2 ; \quad P_{\text {trend }}=0 \cdot 05\right)$. No associations were detected for the 'Meat, Fat and Sweets' dietary pattern and risk of SLL/CLL, other miscellaneous B-cell lymphomas or T-cell lymphomas (online supplementary material, Supplemental Table 1); however, point estimates for all subtypes were in the direction of an increased risk and no evidence of heterogeneity was detected $(P=0.95)$ across the NHL subtypes examined. No associations were detected for the 'Fruit, Vegetables and Starch' dietary pattern and the risk of any of the subtypes examined, nor was there evidence of heterogeneity in the risk estimates $(P=0 \cdot 72)$ across subtypes. Mutual adjustment for the dietary patterns had no effect on the overall or subtype-specific risk estimates (data not shown). 
Table 3 Factor loading matrix for the major dietary patterns identified using principal component factor analysis

\begin{tabular}{lcc}
\hline & Factor 1 & Factor 2 \\
\cline { 2 - 3 } Food or food group & $\begin{array}{c}\text { 'Meat, Fat and Sweets' } \\
\text { dietary pattern }\end{array}$ & $\begin{array}{c}\text { 'Fruit, Vegetables and Starch' } \\
\text { dietary pattern }\end{array}$ \\
\hline French fries & $0 \cdot 71$ & - \\
Red meat & $0 \cdot 70$ & - \\
Processed meat & $0 \cdot 65$ & - \\
Pizza & $0 \cdot 57$ & - \\
Salty snacks & $0 \cdot 57$ & - \\
Sweets and desserts & $0 \cdot 55$ & - \\
Sweetened beverages & $0 \cdot 48$ & - \\
Condiments & $0 \cdot 47$ & - \\
Cooking fat and gravy & $0 \cdot 47$ & - \\
Margarine & $0 \cdot 45$ & - \\
High-fat dairy products & $0 \cdot 39$ & - \\
Pasta and rice & $0 \cdot 38$ & - \\
Eggs & $0 \cdot 37$ & $0 \cdot 68$ \\
Other vegetables & - & $0 \cdot 62$ \\
Tomatoes and juice & - & $0 \cdot 62$ \\
Cruciferous vegetables & - & $0 \cdot 61$ \\
Green leafy vegetables & - & $0 \cdot 57$ \\
Fruit and fruit juice & - & $0 \cdot 56$ \\
Legumes and soya & - & $0 \cdot 50$ \\
Vegetable soup & - & $0 \cdot 47$ \\
Fish & - & $0 \cdot 45$ \\
Mayonnaise or creamy salad dressing & - & $0 \cdot 43$ \\
Cereals and starches & - & $0 \cdot 39$ \\
Potatoes & - & $0 \cdot 33$ \\
Other soups & - & $0 \cdot 31$ \\
Low-fat dairy products & - & - \\
\hline
\end{tabular}

The factor score provides an estimate of the degree to which an individual's diet adheres to the dietary pattern, with higher scores denoting greater adherence. Foods or food groups with factor loadings $<0.30$ on both factors are not presented.

Table 4 Odds ratios and $95 \%$ confidence intervals for non-Hodgkin lymphoma (NHL) according to quartiles of the dietary pattern scores, Nebraska, USA, 1999-2002

\begin{tabular}{|c|c|c|c|c|c|c|c|c|c|c|c|}
\hline & \multirow[b]{2}{*}{ Controls } & \multicolumn{3}{|c|}{$\begin{array}{l}\text { Overall } \\
\mathrm{NHL}\end{array}$} & \multicolumn{3}{|c|}{$\begin{array}{l}\text { Follicular } \\
\text { lymphoma }\end{array}$} & \multicolumn{3}{|c|}{$\begin{array}{c}\text { Diffuse large } \\
\text { B-cell lymphoma }\end{array}$} & \multirow[b]{2}{*}{$P_{\text {heterogeneity }}$} \\
\hline & & Cases & $\mathrm{OR}^{*}$ & $95 \% \mathrm{Cl}$ & Cases & $\mathrm{OR}^{*}$ & $95 \% \mathrm{Cl}$ & Cases & $\mathrm{OR}^{*}$ & $95 \% \mathrm{Cl}$ & \\
\hline \multicolumn{12}{|l|}{$\begin{array}{l}\text { 'Meat, Fat and Sweets' } \\
\text { dietary pattern }\end{array}$} \\
\hline Quartile 1 & 115 & 40 & $1 \cdot 0$ & - & 15 & $1 \cdot 0$ & - & 10 & $1 \cdot 0$ & - & \\
\hline Quartile 2 & 115 & 102 & $2 \cdot 9$ & $1 \cdot 8,4 \cdot 6$ & 25 & 1.9 & $0.9,3.8$ & 29 & $3 \cdot 1$ & $1 \cdot 4,6 \cdot 8$ & \\
\hline Quartile 3 & 115 & 92 & $2 \cdot 7$ & $1 \cdot 7,4 \cdot 5$ & 34 & $2 \cdot 8$ & $1 \cdot 4,5 \cdot 9$ & 21 & $2 \cdot 2$ & $0.9,5.3$ & \\
\hline Quartile 4 & 115 & 99 & $3 \cdot 6$ & $1 \cdot 9,6 \cdot 8$ & 30 & $3 \cdot 1$ & $1 \cdot 2,8 \cdot 0$ & 28 & $3 \cdot 2$ & $1 \cdot 1,9 \cdot 0$ & \\
\hline $\begin{array}{l}P_{\text {trend }} \neq \\
\text { 'Fruit, Vegetables and Starch' } \\
\text { dietary pattern }\end{array}$ & & & $<0.01$ & & & 0.01 & & & 0.09 & & 0.95 \\
\hline Quartile 1 & 115 & 76 & $1 \cdot 0$ & - & 27 & $1 \cdot 0$ & - & 22 & $1 \cdot 0$ & - & \\
\hline Quartile 2 & 115 & 84 & $1 \cdot 1$ & $0.7,1.6$ & 23 & 0.8 & $0.4,1.5$ & 25 & $1 \cdot 1$ & $0.6,2.0$ & \\
\hline Quartile 3 & 115 & 97 & $1 \cdot 2$ & $0 \cdot 8,1 \cdot 8$ & 31 & $1 \cdot 0$ & $0.6,1.9$ & 19 & 0.7 & $0.4,1.5$ & \\
\hline Quartile 4 & 115 & 76 & 0.9 & $0.6,1 \cdot 4$ & 23 & $0 \cdot 7$ & $0.4,1.5$ & 22 & $0 \cdot 7$ & $0.4,1 \cdot 6$ & \\
\hline$P_{\text {trend }} \ddagger$ & & & $0 \cdot 75$ & & & 0.57 & & & $0 \cdot 30$ & & $0 \cdot 72$ \\
\hline
\end{tabular}

*Odds ratios estimated from unconditional logistic regression and adjusted for age (continuous), sex, education ( $<12$ years, $12-15$ years, $16+$ years) and total energy intake (continuous).

$+P$ value for the test of heterogeneity in the parameter estimates across the six $\mathrm{NHL}$ subtypes.

$\ddagger P$ value for the Wald $\chi^{2}$ test of $\mathrm{H}_{\mathrm{o}}: \beta=0$ when modelling quartiles as an ordinal variable.

The associations for the dietary patterns with overall NHL risk were similar in models stratified by sex, age, BMI, smoking status and alcohol consumption and no heterogeneity in the risk estimates was detected for the cross-product terms (data not shown).

\section{Discussion}

In the present population-based case-control study conducted in Nebraska, we identified a 'Meat Fat, and Sweets' dietary pattern and a 'Fruit, Vegetables and Starch' dietary 
pattern using principal component factor extraction. In multivariable models, the 'Meat, Fat and Sweets' dietary pattern was associated with an increased risk of overall NHL. In secondary analyses stratified by NHL subtype, the 'Meat, Fat and Sweets' dietary pattern was associated with an increased risk of FL, DLBCL and MZL. No association with overall or subtype-specific NHL risk was detected for the 'Fruit, Vegetables and Starch' dietary pattern.

To the best of our knowledge, only one prior study has examined dietary patterns in relation to the risk of NHL. In a multiethnic cohort of older adults, Erber et al. ${ }^{(16)}$ reported no association with NHL risk for a 'Meat and Fat' dietary pattern characterized by high intakes of discretionary fat, meat and organ meat, processed meat, white potatoes, non-whole grains, eggs and cheese. However, the association for this 'Meat and Fat' dietary pattern was in the direction of an increased risk $\left(\mathrm{OR}_{\mathrm{T} 3} v\right.$. T1 $=1 \cdot 40$, $95 \%$ CI $0 \cdot 82,2 \cdot 41$ ) among Caucasian males. In our study, the association between the 'Meat, Fat and Sweets' dietary pattern and overall NHL risk was similar for men and women. Discrepancies in the findings between these studies may reflect differences in the individual food items aggregated and entered into the factor analysis based, in part, on differences in the location, age distribution and ethnic composition of the study participants. In addition, the 'Meat, Fat and Sweets' dietary pattern identified in our sample also loaded high on sweets and desserts, sweetened beverages, and pasta and rice (rich sources of refined carbohydrates and other nutrients), as well as high-fat dairy products which may have influenced the results. Differences in the study designs, including the potential for recall bias in our case-control study, may have also contributed to the discrepant findings. In addition, to the extent to which unmeasured changes in dietary intake occurred over the follow-up period, misclassification error may have attenuated the associations reported for the Multiethnic Cohort (MEC) study. In line with our results, no associations with overall NHL risk were detected in the MEC study for a dietary pattern characterized by high intakes of fruits and vegetables or for a dietary pattern characterized by high intakes of milk and yoghurt and fruits ${ }^{(16)}$.

In the secondary analyses examining the risk of specific NHL subtypes, the 'Meat, Fat and Sweets' dietary pattern was associated with an increased risk of FL, DLBCL and MZL, with the point estimates for all subtypes in the direction of an increased risk. In the MEC study, the risk of FL was increased fivefold among males, but not females, with the highest factor scores for the 'Meat and Fat' dietary pattern ${ }^{(16)}$. In our sample, we detected no heterogeneity in the association between the 'Meat, Fat and Sweets' dietary pattern and the risk of FL by sex. In agreement with our results, no consistent association with the risk of FL, DLBCL or SLL/CLL was detected for a dietary pattern characterized by high intakes of fruits and vegetables, or for a dietary pattern characterized by high intakes of milk and yoghurt and fruits, in the MEC study ${ }^{(16)}$.

Dietary pattern analysis addresses several limitations of traditional approaches examining associations of single foods or nutrients in relation to cancer risk. Factor analysis provides an empirically derived measure that better reflects the combination of foods consumed ad libitum in the diet of free-living people, accounts for potential interactions between foods and nutrients, aggregates additive effects of single items included in the composite factor, provides a measure more amenable to dietary recommendations, and accounts for intercorrelations between foods and/or nutrients ${ }^{(7)}$. However, dietary pattern analysis is not without its limitations. Factor analysis requires subjective decisions to be made at several points when deriving dietary patterns ${ }^{(7)}$, may provide little insight into the biological processes underlying the associations with disease risk ${ }^{(17)}$, and empirically derived dietary patterns may not be reproducible across populations.

The current study has several strengths including the confirmation of NHL diagnoses by an expert haematopathologist; the high response rates for cases (73\%) and controls (77\%); the recruitment of randomly selected, population-based controls sampled from the same source population giving rise to the cases; the use of a rapid case ascertainment system to minimize the potential for survival bias; and the use of a validated FFQ. There were also limitations. First, as previously discussed, our factor analysis attempting to identify empirically derived, a posteriori dietary patterns required subjective decisions regarding the classification of food groups and the retention of factors. To address this limitation, we adhered closely to previous studies when aggregating food items into predefined groups $^{(7)}$ and conducted a second factor analysis on all 117 food items to assess the influence of the classification on the dietary patterns identified. In addition, we closely followed established guidelines with respect to the retention of factors ${ }^{(14)}$ and identified a 'Meat, Fat and Sweets' dietary pattern and a 'Fruit, Vegetables and Starch' dietary pattern similar to those reported in previous studies ${ }^{(7)}$, providing support as to their reproducibility in other populations. Second, the dietary patterns retained in our analysis explained only a small portion of the total variance (8.6\%). Third, the small number cases limited our ability to detect associations in subtype-specific analyses, as well as our ability to detect heterogeneity in the risk estimates across the NHL subtypes examined. Fourth, we cannot rule out the possibility of residual confounding and recall bias.

\section{Conclusion}

Our findings suggest that greater adherence to a 'Meat, Fat and Sweets' dietary pattern may increase the risk of NHL. Additional studies, ideally allowing for prediagnostic exposure assessment, are required to confirm these findings. 
In addition, future studies should consider whether associations between dietary patterns and the risk of NHL are consistent across racial or ethnic groups. The associations detected for specific NHL subtypes in our population also highlight the need for pooled analyses of NHL subtypes to address aetiological heterogeneity.

\section{Acknowledgements}

Sources of funding: This research was supported by research grant 99B083 from the American Institute for Cancer Research and, in part, by grants CA94770 and CA100555 from the National Cancer Institute. Conflicts of interest: The authors have no conflicts of interest to declare. Authors' contributions: N.J.O. designed and carried out the statistical analysis, interpreted the results and wrote the manuscript; B.A.-K. provided insight into the analytic strategy and interpretation of results; D.B.C.C. provided insight into the analytic strategy and interpretation of results; S.M.S. provided insight into the analytic strategy and interpretation of results; D.D.W. conducted the pathology review, provided insight into the analytic strategy and interpretation of results; B.C.-H.C. collected the data, originally conceived of the analysis, provided insight into the analytic strategy and interpreted the results. All authors provided feedback on the initial draft of the manuscript and approved the final version. Acknowledgements: The authors thank Mr Martin Bast of the Nebraska Lymphoma Study Group for coordinating the case identification and for obtaining the physician consents for the study cases.

\section{Supplementary material}

To view supplementary material for this article, please visit http://dx.doi.org/10.1017/S1368980013001249

\section{References}

1. Siegel R, Ward E, Brawley O et al. (2011) Cancer statistics, 2011: the impact of eliminating socioeconomic and racial disparities on premature cancer deaths. CA Cancer J Clin 61, 212-236.

2. Hennessy BT, Hanrahan EO \& Daly PA (2004) NonHodgkin lymphoma: an update. Lancet Oncol 5, 341-353.

3. Ekstrom-Smedby K (2006) Epidemiology and etiology of non-Hodgkin lymphoma - a review. Acta Oncol 45, 258-271.

4. Alexander DD, Mink PJ, Adami HO et al. (2007) The non-Hodgkin lymphomas: a review of the epidemiologic literature. Int J Cancer 120, Suppl. 12, 1-39.

5. Skibola CF (2007) Obesity, diet and risk of non-Hodgkin lymphoma. Cancer Epidemiol Biomarkers Prev 16, 392-395.

6. Cross AJ \& Lim U (2006) The role of dietary factors in the epidemiology of non-Hodgkin's lymphoma. Leuk Lymphoma 47, 2477-2487.

7. Newby PK \& Tucker KL (2004) Empirically derived eating patterns using factor or cluster analysis: a review. Nutr Rev 62, 177-203.

8. Chiu BC, Kolar C, Gapstur SM et al. (2005) Association of NAT and GST polymorphisms with non-Hodgkin's lymphoma: a population-based case-control study. Br J Haematol 128, 610-615.

9. Chiu BC, Soni L, Gapstur SM et al. (2007) Obesity and risk of non-Hodgkin lymphoma (United States). Cancer Causes Control 18, 677-685.

10. Jaffe ES, Harris NL, Stein H et al. (editors) (2001) World Health Organization Classification of Tumors. Pathology and Genetics of Tumors of Hematopoietic and Lymphoid Tissues. Lyon: IARC Press.

11. Block G, Coyle LM, Hartman AM et al. (1994) Revision of dietary analysis software for the Health Habits and History Questionnaire. Am J Epidemiol 139, 1190-1196.

12. Block G, Hartman AM, Dresser CM et al. (1986) A databased approach to diet questionnaire design and testing. Am J Epidemiol 124, 453-469.

13. Block G, Woods M, Potosky A et al. (1990) Validation of a self-administered diet history questionnaire using multiple diet records. J Clin Epidemiol 43, 1327-1335.

14. Kim J-O \& Mueller CW (1978) Introduction to Factor Analysis. Newbury Park, CA: SAGE Publications.

15. Mickey RM \& Greenland S (1989) The impact of confounder selection criteria on effect estimation. Am J Epidemiol 129, 125-137.

16. Erber E, Maskarinec G, Gill JK et al. (2009) Dietary patterns and the risk of non-Hodgkin lymphoma: the Multiethnic Cohort. Leuk Lymphoma 50, 1269-1275.

17. Hu FB, Rimm EB, Stampfer MJ et al. (2000) Prospective study of major dietary patterns and risk of coronary heart disease in men. Am J Clin Nutr 72, 912-921. 